DOI: https://doi.org/10.15407/mzu2020.29.130

UDC 930.1[493+435.9]

\title{
Aliesia Soloviova
}

étudiante diplômée

l'Institut de l'histoire de l'Ukraine

l'Académie nationale des sciences de l'Ukraine

4, rue Mykhajla Hrushevs'koho, Kyiv 01001, Ukraine

E-mail: aliesia.soloviova@gmail.com

ORCID: https://orcid.org/0000-0001-8384-0512

\section{LE RÔLE DU FACTEUR RUSSE DANS L'ATTITUDE DES PAYS DU BENELUX ENVERS L'UKRAINE (2014-2019)}

L'auteur de cet article a réalisé une analyse du rôle du facteur russe dans l'attitude des pays du Benelux envers l'Ukraine de 2014 à 2019. L'analyse a montré qu'il est extrêmement important à ce stade de comprendre l'influence de la Russie sur les relations entre l'Ukraine et les pays du Benelux. Cette approche permet de déterminer les raisons de la stagnation éventuelle de ces relations et du dialogue entre États.

En général, les stratégies des trois pays du Benelux peuvent être qualifiées de pragmatiques. Cette approche s'explique, tout d'abord, par une coopération étroite dans le domaine de l'économie et les inconvénients d'une rupture complète des relations avec la Russie. Les pays du Benelux sont de petits pays par rapport à leurs voisins européens, dont la principale force est la stabilité économique. Ainsi, pour les pays du Benelux, une intervention active dans le conflit Russie-Ukraine constitue une menace pour les relations économiques.

Dans le même temps, les pays du Benelux soutiennent généralement la politique de sanctions de l'Union européenne contre la Russie, mais insistent sur le dialogue, considérant les sanctions comme un moyen moins efficace d'atteindre les objectifs. Les Pays-Bas ont la position la plus active parmi les pays du Benelux dans le conflit armé russo-ukrainien, cependant, cette position est principalement due à des préoccupations internes et à l'enquête en cours sur la chute du Boeing MH17 sur le territoire ukrainien.

Mots clefs: politique étrangère, l'intégration européenne, les pays du Benelux, la politique de l'Union européenne. 


\section{Aliesia Soloviova}

Postgraduate Student

Institute of History of Ukraine

the National Academy of Sciences of Ukraine

4, Mykhailo Hrushevskyi Street, Kyiv, 01001, Ukraine

E-mail: aliesia.soloviova@gmail.com

ORCID: https://orcid.org/0000-0001-8384-0512

\section{THE ROLE OF THE RUSSIAN FACTOR IN THE ATTITUDE OF BENELUX COUNTRIES TOWARDS UKRAINE (2014-2019)}

The article analyzes the role of the Russian factor in the attitude of Benelux countries towards Ukraine from 2014 to 2019. The author proceeds from the fact that one of the factors in a state's choice of a certain strategy in the international arena is often the size of the state. The study of the place and role of "small" states has become a separate field of study in the history of international relations since the publication of the work of the American researcher Annette Baker. Proponents of this theory share the opinion that the size of a state's territory significantly affects its political strategy. The author notes that there is no single definition of a "small" state, since the characteristics by which the state as such is defined are constantly changing. In general, the signs of a "small" state are small population, size of territory, low level of activity in the international arena.

The author of the article concludes that the foreign policy strategies of all three Benelux countries can be called pragmatic. In the author's opinion, this approach is explained, first of all, by close cooperation in the field of economics and disadvantage of completely breaking off relations with Russia. The Benelux countries are small states compared to their European neighbors; their main strength is economic stability. It was found that for the Benelux countries, active intervention in the conflict between Russia and Ukraine means a threat to economic ties. The Benelux countries generally support the EU's sanctions policy, but insist on dialogue, considering sanctions a fewer effective means of achieving goals. The Netherlands occupies the most active position among the Benelux countries in the conflict, however, this position is mainly caused by internal concerns and the ongoing investigation of the Boeing MH17 crash.

Keywords: foreign policy, European integration, Benelux countries, European Union policy.

Dans le contexte du vecteur de la politique étrangère de l'Ukraine d'intégration au sein de l'Union européenne, compte tenu de la position 
géographique de l'État ukrainien, les études sur les perspectives de cette intégration de l'Ukraine deviennent de plus en plus importantes. Mais, comme le souligne le chercheur bien connu de l'histoire de la diplomatie ukrainienne Mycola Doroshko, "si pour un certain nombre d'États post-socialistes qui sont les membres à part entière actuels de la communauté européenne, les principaux critères d'adhésion à l'UE étaient des indicateurs de croissance économique et un mouvement cohérent à travers la démocratisation de la société, néanmoins les relations ukraino-russes ont un impact énorme sur les perspectives d'intégration européenne de l'Ukraine ${ }^{1 "}$. À notre avis, le dialogue politique entre l'Ukraine et l'Union européenne (ci-après dénommée l'UE), ainsi que l'Ukraine et les pays du Benelux dépend d'un certain nombre de facteurs, parmi lesquels le facteur russe est l'un des facteurs déterminants. Dans ce contexte, il est particulièrement important d'analyser le développements de l'intégration européenne de l'Ukraine et les relations UkraineBenelux à travers le prisme du facteur russe, ainsi que de suivre les prochaines mesures de l'Ukraine dans le processus d'approche de l'UE et dans les relations Ukraine-Benelux.

Les études de problèmes et de perspectives sur l'intégration européenne de l'Ukraine ont été menée par des scientifiques tels que: O.O. Akimov, I.V. Artomov, V.D. Bogdanovych, N.M. Buglaj, S.V. Vidnyanskyj, L.V. Vitrenko, O.S. Vlasyuk, V.P. Gorbulin, O.M. Gorenko, Ye.O. Goryunova, M.S. Doroshko, I.A. Grycyak, B.I. Kancelyaruk, V.V. Kopijka, E.M. Libanova, O.M. Lyashenko, A.Yu. Martynov, P.P. Gaj-Nyzhnyk, L.G. Olejnikova, G.M. Perepelycya, O.M. Sheretyuk et autres. Parmi les travaux qui sont directement consacrés aux principales étapes et problèmes des relations Ukraine-Union européenne, il convient de noter les travaux de S.V. Vidnyanskyj, qui dans la chronologie des relations entre l'Ukraine et l'UE a identifié et décrit 5 étapes, mettant en évidence la période actuelle - à partir de 2014, associée à la signature, la ratification et la mise en œuvre de l'accord d'association entre l'Ukraine et l'Union européenne dans des conditions de confrontation militaire dans l'est de l'Ukraine, de crise de l'Etat ukrainien, de crise systémique et début de la réforme de l'UE elle-même ${ }^{2}$.

L'un des premiers ouvrages publiés sur les particularités de la politique étrangère des États qui ne sont pas de grande taille, de population, etc., a été le travail du chercheur américain A. Baker Fox "The power of small states: diplomacy in World War II". L'étude de la théorie selon laquelle la taille du territoire de l'État affecte de manière significative sa stratégie politique a été entreprise par des chercheurs étrangers tels que: I.Karsh, O. Knudsen, B. Torhallsson, S. Steinsson et autres. Des chercheurs européens tels que L. Mark, $\mathrm{N}$. Popescu et d'autres ont étudié les stratégies poursuivies par les États membres de l'UE en relation avec la Russie. 
Le but de cet article est de révéler le rôle du facteur russe dans l'attitude des pays du Benelux envers l'Ukraine de 2014 à 2019.

La stratégie de sécurité de l'UE de 2003 a désigné la Russie comme un acteur majeur sur le plan géopolitique, tant au niveau mondial que régional. Comme indiqué dans ce document, la Russie est un marché important et dynamique pour les biens et services de l'UE avec une croissance économique significative. D'un autre côté, le marché de l'UE est de loin la destination la plus importante pour les exportations russes. Les entreprises de l'UE sont d'importants investisseurs en Russie ${ }^{3}$. Mykola Doroshko note que la Russie considère la région de la Communauté d'États indépendants (CEI) uniquement comme une zone d'influence politique. Dans ce contexte, la vision géostratégique de la Russie envisage l'adhésion de l'Ukraine aux alliances dans l'espace post-soviétique sous les auspices de la Fédération de Russie, ce qui contredit le choix stratégique européen de l'Ukraine ${ }^{4}$.

Olga Sheretyuk attire l'attention sur le fait qu'en Ukraine, malgré les déclarations officielles sur l'intégration européenne en tant que priorité de politique étrangère, l'élite politique ne fait pas toujours preuve de cohérence dans la mise en œuvre d'un certain cours dans la politique étrangère ${ }^{5}$. L'auteure note que le succès de la stratégie d'intégration européenne dépendra de la mise en œuvre des règles de l'UE et des relations avec la Russie, la Biélorussie, la Moldavie et d'autres pays post-soviétiques ${ }^{6}$.

Les événements survenus en Ukraine en 2014 ont eu un impact significatif sur les relations UE-Russie, cependant, les États membres de l'UE prennent des positions différentes sur le conflit existant entre l'Ukraine et la Russie, et définissent différentes stratégies politiques pour résoudre le conflit et même pour poursuivre la coopération avec la Russie.

En 2017, une étude soutenue par le Parti populaire européen au Parlement européen a été réalisée. L'étude s'intitulait "Comment les démocraties européennes réagissent-elles à l'agression russe?" ("How do European democracies react to Russian aggression?"). L'étude a révélé qu'il existe plusieurs stratégies sélectionnées par les pays de l'UE pour les relations avec la Russie après l'introduction de sanctions, à savoir: 1) six pays qui prennent une position active en réponse à l'agression russe (Estonie, Lettonie, Lituanie, Pologne, Grande-Bretagne, Danemark); 2) cinq pays qui ont considérablement modifié leur politique à l'égard de la Fédération de Russie après l'agression russe contre l'Ukraine (Finlande, Suède, Pays-Bas, République Tchèque, Allemagne); 3) trois pays sont partisans de la lutte contre l'agression russe (Croatie, Roumanie, Bulgarie); 4) trois Etats n'ont pratiquement aucune relation avec la Russie (Portugal, Malte, Irlande); 5) six pays tentent de suivre les problèmes existants (Autriche, Belgique, France, Luxembourg, Espagne, Slovénie); 6) deux gouvernements utilisent la carte russe pour des raisons internes 
(Slovaquie, Hongrie); 7) trois états ont une attitude positive envers le Kremlin (Grèce, Italie, Chypre) ${ }^{7}$. Il convient de noter qu'en octobre 2016, des scientifiques ukrainiens ont divisé les États membres de l'UE en trois groupes : les dirigeants soutenant l'Ukraine, les observateurs et les opposants à l'Ukraine ${ }^{8}$.

Les chercheurs européens Leonard Mark et Nick Popescu ont identifié cinq types de stratégies politiques mises en œuvre par les États membres de l'UE vis-à-vis de la Russie. Ils ont notamment identifié les stratégies suivantes: 1) un cheval de Troie (les États défendent des positions proches des intérêts russes et qui sont prêts à opposer leur veto à des positions communes de l'UE); 2) les partenaires stratégiques (les États qui ont des "relations spéciales" avec la Russie, qui affectent périodiquement la politique globale de l'UE); 3) le pragmatisme amical (les États qui entretiennent des relations étroites avec la Russie et, en règle générale, mettent leurs intérêts commerciaux au-dessus des objectifs politiques); 4) le pragmatisme froid (les pays qui se concentrent également sur les intérêts des entreprises mais n'ont pas "peur" de dénoncer la politique de la Russie); et 5) les nouveaux guerriers froids (les pays qui ont des relations ouvertement hostiles avec Moscou et sont prêts à utiliser le veto pour bloquer les négociations de l'UE avec la Russie) ${ }^{9}$. Selon cette classification, les pays du Benelux appartiennent à la catégorie des "pragmatistes", le Luxembourg et la Belgique ont tendance à "un pragmatisme amical", et les Pays-Bas, à leur tour, ont soi-disant "un pragmatisme froid". La présence d'une telle variété de stratégies des États membres de l'UE pour les relations avec la Russie nécessite d'explorer les raisons du choix de la politique étrangère.

Le choix de la stratégie sur la scène internationale est souvent produit par la taille de l'État. Les études sur les petits États sont devenues un domaine d'étude distinct dans les relations internationales depuis la publication des travaux de la chercheuse américaine Annette May Baker Fox sur la politique des "petits" États pendant la Seconde Guerre mondiale en $1959^{10,11}$. Les partisans de cette théorie partagent le point de vue selon lequel la taille du territoire de l'État influe considérablement sur sa stratégie politique.

Il n'y a pas de définition unique d'un "petit" État, car les signes qui définissent l'État en tant que tel changent constamment. En général, les signes d'un "petit" État sont les suivants : la petite population et taille du territoire, le faible niveau d'activité sur la scène internationale.

Le professeur Baldur Thorhallsson de l'Université d'Islande propose de définir les "petits" États selon les critères suivants: la taille (la population et le territoire), la souveraineté (le degré de contrôle de l'état de ses affaires intérieures et de ses frontières), autorité politique (potentiel militaire, cohésion interne), le développement économique (PIB, taille du marché et développement), les perceptions sur la scène internationale et les avantages (les idées, 
ambitions et priorités de l'élite interne concernant leur rôle dans le système international) $)^{12}$.

Les petits États sont géographiquement et économiquement diversifiés et sont donc confrontés à des défis de sécurité et de bien-être différents. Par exemple, le luxueux Luxembourg, l'État membre fondateur de l'Union européenne, qui occupe une position centrale dans la politique européenne, est confronté à des problèmes autres que les pays baltes. À leur tour, les problèmes rencontrés par des États tels que les États baltes et le Luxembourg diffèrent des défis de la République centrafricaine ou du Libéria, où règne la pauvreté ${ }^{13}$.

Parallèlement à la question de la définition de tels États, une attention particulière est accordée aux particularités de la politique étrangère de ces États. La question de la protection des frontières et des populations contre d'éventuelles menaces internationales et le rôle des "petits" États sur la scène internationale sont particulièrement importants.

Le Luxembourg a longtemps été le plus petit État membre de l'UE, mais après l'élargissement de 2004, Malte a pris sa place. Au siècle dernier, la situation géographique du Luxembourg a aidé le pays à jouer le rôle de médiateur influent entre les anciens opposants - l'Allemagne et la France. Selon des chercheurs étrangers, outre la situation géographique, l'influence du Luxembourg s'explique par trois autres facteurs : l'innovation, la stabilité et une "bonne coïncidence". Bien qu'il soit difficile d'être d'accord avec ce dernier, les deux autres facteurs semblent assez logiques. La stabilité politique a grandement contribué à l'évolution du pays. Pierre Werner a travaillé au gouvernement depuis 25 ans et pendant 20 ans a occupé le poste de chef du gouvernement. Pierre Werner a rejoint le gouvernement luxembourgeois en tant que ministre des Finances en 1953 et a été Premier ministre de 1959 à 1974 et de 1979 à 1984. À son tour, Jacques Santer a été 17 ans au gouvernement, 11 ans comme Premier ministre et 10 ans au Parlement européen. En 1984, suite à la démission de Pierre Werner, Jacques Santer devient le nouveau Premier ministre luxembourgeois, demeurant ministre luxembourgeois des Finances jusqu'en 1989. En outre, de 1984 à 1989 il est gouverneur de la Banque mondiale, de 1989 à 1994 il est gouverneur du Fonds monétaire international. En 1995, Jacques Santer a démissionné de son poste de Premier ministre luxembourgeois pour devenir président de la Commission européenne. Jean-Claude Juncker a servi le gouvernement pendant 25 ans, dont 12 ans en tant que Premier ministre. En général, le pays a toujours cherché à maintenir une politique intérieure stable grâce à une administration efficace.

Outre le rôle joué par les "petits" États sur la scène internationale, la question des caractéristiques inhérentes à leur politique étrangère est également importante. De nombreux chercheurs, dont Efraim Karsh, affirment que la neutralité est une caractéristique déterminante de nombreux petits États. La 
"survie" d'un petit État dépend souvent de sa capacité à démontrer qu'il ne constitue pas une menace pour les grandes puissances ${ }^{14}$. Dans ce contexte, l'attitude du Luxembourg à l'égard du conflit russo-ukrainien en cours et l'implication de l'UE, à savoir l'attitude du pays vis-à-vis des sanctions européennes imposées à la Russie pour ingérence dans les affaires ukrainiennes, sont intéressantes à cet égard. Dans cette affaire, le Grand-Duché était et reste neutre, probablement parce que le Luxembourg est (au moins sur papier) le troisième investisseur étranger en Russie et un grand centre financier pour les banques et les entreprises russes ${ }^{15}$.

Le Premier ministre luxembourgeois a une position assez neutre sur le conflit. Dans une interview accordée à EURACTIV, Xavier Bethel a déclaré en répondant à des questions sur les raisons du non-respect des accords de Minsk: "Les chefs d'État devraient respecter l'accord signé. Les deux parties ont commis des erreurs. Je ne veux pas rejeter la faute sur un seul côté. Il est important pour moi de comprendre comment nous pouvons avancer, car pour le moment nous sommes tous dans une situation perdante. L'Europe perd, l'Ukraine perd, la Russie perd. Nous avons besoin d'une stratégie de sortie pour trouver des solutions avec lesquelles tout le monde peut vivre" ${ }^{\text {"16 }}$. Cette approche n'est pas nouvelle au Luxembourg. L'attitude pragmatique face aux conflits externes est illustrée par la déclaration du ministre luxembourgeois des Affaires étrangères Jean Asselborn en 2009 : "La Russie n'a pas de droit de veto sur le choix de pays comme la Géorgie et l'Ukraine. Cependant, il est important de prendre en compte les préoccupations de la Russie afin d'éviter la menace inutile de stabilité et d'équilibre politique dans notre région. L'avantage d'un partenariat stratégique basé sur la confiance mutuelle est que les différences peuvent être abordées de manière ouverte et constructive" ${ }^{\text {"17 }}$.

Les partenariats commerciaux et les orientations économiques des "petits" États, en particulier de Luxembourg, ont une influence significative sur le choix de la politique étrangère. Le Luxembourg est l'un des pays les plus dépendants des importations d'énergie. Environ $60 \%$ des besoins énergétiques du pays sont couverts par les produits pétroliers. Le gaz naturel est la deuxième forme d'énergie la plus importante. Les produits pétroliers proviennent des pays voisins, principalement de Belgique (77,2\% en 2013). Alors qu'en 2013, la Russie représentait $24 \%$ des importations de gaz naturel au Luxembourg ${ }^{18}$. Dans le grand secteur financier luxembourgeois, il existe des investissements étrangers directs et des investissements de portefeuille en provenance de Russie, qui représentent plus de $40 \%$ du PIB, ses investissements sont beaucoup plus importants en Russie, équivalant à $120 \%$ du PIB du Luxembourg ${ }^{19}$.

La Russie et le Luxembourg continuent de coopérer activement dans le domaine de l'économie. Le 18 février 2016, la dixième session de la Commission mixte de coopération économique de l'Union économique belgo- 
luxembourgeoise et la Russie (UEBL-Russie) a eu lieu, en présence du vicePremier ministre Dmitry Rogozin, vice-Premier ministre du Grand-Duché de Luxembourg Etienne Schneider et Ambassadeur du Royaume de Belgique auprès de la Fédération de Russie Alex Van Mioven. Etienne Schneider était accompagné d'une délégation de représentants de la Chambre de commerce du Luxembourg. Au sein de cette commission mixte, la Chambre de commerce et d'industrie a organisé conjointement avec des organisateurs russes un forum d'affaires sur "l'investissement et l'innovation" et "le transport et la logistique". La Commission mixte UEBL-Russie a signé un protocole d'accord entre l'Agence nationale de recherche et d'innovation du Luxembourg et le Centre russe de Skolkovo pour renforcer la coopération en matière d'innovation et de nouvelles technologies ${ }^{20}$.

Le Luxembourg, bien sûr, continue de considérer la Russie comme un partenaire stratégique important et soutient donc une approche plus souple de la question des sanctions. Le Luxembourg, dans son ensemble, soutient la politique générale de l'UE consistant à imposer des sanctions à la Russie pour violation du droit international. Le Luxembourg considère les sanctions comme un instrument d'influence inefficace au motif qu'elles se sont révélées inefficaces pour atteindre cet objectif.

En 1974, Alfred van Staden, professeur de relations internationales à l'Université de Leiden (les Pays-Bas), dans son étude "L'Allié fidèle : les Pays-Bas et l'Alliance atlantique (1960-1971)" ("A Faithfull ally: The Netherlands and the Atlantic Alliance (1960-1971)") a décrit la politique étrangère des Pays-Bas comme "réactive plutôt qu'active. En général, les gouvernements [néerlandais] s'assoient sur la clôture et ne répondent qu'aux rafales extérieures" ${ }^{\prime 2}$. Cette déclaration, dans son ensemble, reste pertinente pour la politique actuelle des Pays-Bas. Le gouvernement néerlandais est dominé par l'idée que, la Russie et les Pays-Bas partageant de nombreux intérêts, la tâche est de continuer à coopérer. Dans le même temps, les PaysBas ne veulent pas donner l'impression qu'il est possible de continuer à faire des affaires comme d'habitude (business as usual) pendant que la Russie continue de s'ingérer dans les affaires ukrainiennes. Le principal représentant des intérêts commerciaux, Hans de Boer, président de la Confédération néerlandaise des entreprises et des employeurs VNO-NCW, a exprimé des doutes quant à l'efficacité des sanctions et a dit craindre que la poursuite des sanctions aide les entreprises brésiliennes et chinoises à conquérir le marché russe au détriment des intérêts néerlandais ${ }^{22}$.

Le chercheur russe Andrey Makarov note que bien que "depuis l'introduction des sanctions de l'UE contre la Russie le 17 mars 2014, et après le 17 juin 2014, lorsque le crash de l'avion avec le Boeing malaisien s'est produit, le dialogue intergouvernemental bilatéral a été essentiellement gelé 
jusqu'à présent" 23 , mais "malgré des facteurs externes, la Russie et les PaysBas sont toujours restés des partenaires importants dans le domaine du commerce et de l'investissement" 24 .

Actuellement, le Royaume des Pays-Bas pour la Fédération de Russie est l'un des principaux fournisseurs de biens, services, compétences et technologies dans pratiquement tous les secteurs de l'économie, pays de transit et plaque tournante logistique pour les exportations russes, ainsi qu'un partenaire important dans le domaine des investissements transfrontaliers. Les Pays-Bas est traditionnellement le plus grand partenaire commercial de la Russie (3ème place au monde après la Chine et l'Allemagne, 2ème en Europe après l'Allemagne), leur part dans le chiffre d'affaires total de la Fédération de Russie est stable à $6-7 \%{ }^{25}$. En 2017, selon le Service fédéral des douanes de Russie, le commerce russo-néerlandais s'est élevé à 39,5 milliards de dollars, en hausse de 22,4\% par rapport aux exportations russes de 2016. Les exportations russes vers les Pays-Bas ont atteint 35,6 milliards de dollars $(+21,7 \%)$ et les importations en provenance des Pays-Bas - 3,0 milliards de dollars $(-3,2 \%)^{26}$.

Malgré l'approche plutôt pragmatique suivie par le Luxembourg et les Pays-Bas, les Pays-Bas sont encore plus préoccupés par l'agression de la Russie. En témoigne la réaction assez rapide aux événements en Ukraine. Dans sa lettre du 12 juin 2014, le ministère des Affaires étrangères des Pays-Bas a pour la première fois clairement appelé l'annexion de la Crimée - une annexion illégale $^{27}$. En outre, le ministère des Affaires étrangères des Pays-Bas a déclaré que l'attitude de la Russie à l'égard de l'Ukraine était inacceptable ("onaanvaardbaar") $)^{28}$.

L'impact le plus négatif sur les relations entre les Pays-Bas et la Russie a été causé par l'écrasement du Malaysia Airlines MH17, qui a tué 196 citoyens néerlandais, ce qui a été un énorme choc pour le public néerlandais. À partir de ce moment, il est devenu clair qu'il ne pouvait pas y avoir de retour rapide au "des affaires comme d'habitude".

En 2015, le ministre des Affaires étrangères des Pays-Bas, Bert Kunders (17 octobre 2014-2017), a décrit une nouvelle approche de la Russie, basée sur une stratégie de "poing fermé" et de "main ouverte". La politique du "poing fermé" signifiait la poursuite de la sanction jusqu'à ce que Moscou commence à travailler à la désescalade du conflit dans le Donbass et / ou à abandonner la Crimée. Ce faisant, les Pays-Bas maintiendront un dialogue avec la Russie en tant que partenaire égal sur la base du respect mutuel. Les Pays-Bas insistent sur le maintien des relations économiques et le maintien de la coopération dans des domaines tels que l'éducation, la science et la société civile ${ }^{29}$. Ceci en dépit du fait que 2015 a été l'année des tentatives de la Russie de mettre en œuvre le scénario de guerre hybride intra-phase à travers le pseudo-pacifique "processus 
de Minsk" soutenu par les pays occidentaux ${ }^{30}$. La Fédération de Russie ne pouvait accepter non seulement sa défaite géopolitique dans la lutte pour une prise de contrôle "douce" de l'Ukraine, mais aussi son existence même en tant qu'État. Par conséquent, le Kremlin a décidé d'utiliser dans la lutte contre l'Ukraine non seulement toutes les méthodes ouvertes et secrètes pour mener une guerre hybride, mais aussi l'ingérence armée directe dans ses affaires intérieures ${ }^{31}$. Burt Kunders a déclaré: "Nous devons continuer de faire pression sur la Russie, mais également rechercher de nouvelles solutions politiques. La Russie et les Russes ne sont pas nos ennemis, mais nous ne pouvons pas permettre l'application unilatérale des règles européennes sur la souveraineté nationale et le recours à la force, ${ }^{, 32}$.

L'actuel ministre des Affaires étrangères des Pays-Bas, Stef Blok (de 2018 à maintenant), a souligné l'urgence de la politique de "pression et dialogue" ("druk en dialogue"). Le gouvernement souligne également que cette politique ne peut réussir que si l'UE et l'OTAN continuent d'agir dans l'unité. Le moyen de pression le plus important est le régime de sanctions imposé par l'UE en réponse aux actions de la Russie contre l'Ukraine. Ces sanctions visent spécifiquement les politiciens russes directement impliqués dans des violations de la souveraineté et de l'intégrité territoriale de l'Ukraine. De plus, traduire en justice les responsables de l'accident du MH17 demeure la plus haute priorité du gouvernement.

En général, les discussions sur le conflit entre l'Ukraine et la Russie ne se sont pas apaisées dans la société néerlandaise. Dans la perspective des élections au Parlement européen, lors d'un débat avec le Premier ministre néerlandais, Mark Rutte (depuis 2010), le leader du parti Forum pour la démocratie, Thierry Bode, s'est prononcé en faveur du retrait du pays de l'Union européenne et du rapprochement avec la Russie. Le sujet des relations avec la Russie n'a pas été ignoré. Bode a déclaré: "Dans la politique internationale, vous n'avez ni amis ni ennemis, seulement des intérêts". Selon Bode, ces intérêts sont lésés, par exemple, par des sanctions contre la Russie, qui affectent l'agriculture et le secteur des fleurs. Le débat s'est intensifié, car Bode a refusé de convenir que la Russie était probablement à l'origine de la chute du vol MH17. "J'ai des doutes quant à l'enquête sur le JIT [une équipe internationale d'enquête sur le désastre du MH17] ${ }^{33}$. L'Ukraine est également l'un des coupables possibles de cette attaque". Selon un sondage De Honds de 2015, la grande majorité des citoyens néerlandais (78\%) pensent que la Russie est directement ou indirectement responsable de l'écrasement du MH17.

Ainsi, le public néerlandais est moins intéressé par une discussion fondamentale sur les relations avec la Russie. La question principale est la question plus large de savoir si les Pays-Bas devraient être activement impliqués dans la coopération internationale. 
En raison de sa situation au carrefour de l'Europe occidentale, la Belgique traditionnellement cherche à éviter la domination des pays les plus puissants qui l'entourent par la médiation.

Avec 6,86 milliards d'euros d'importations belges en provenance de Russie en 2017, la Russie se classe au troisième rang des partenaires commerciaux non européens les plus importants de la Belgique. Parmi les partenaires non européens, la Russie occupe une place relativement importante malgré les sanctions de 2014. Par exemple, en termes d'importations, c'est le troisième partenaire non européen de la Belgique après les États-Unis et la Chine. En ce qui concerne les exportations de la Belgique, la Russie est le cinquième partenaire hors UE après les États-Unis, la Chine, l'Inde et la Turquie $^{34}$.

En 2015, le ministre belge des Affaires étrangères Didier Reinders sur les priorités de la diplomatie belge a noté que la Russie reste un partenaire important, ce qui nécessite le soutien d'un dialogue ouvert. À long terme, il est nécessaire de revoir les relations UE-Russie quelles que soient les sanctions existantes, et de traiter ensemble des questions telles que les droits de l'homme, les relations commerciales, la coopération énergétique, etc ${ }^{35}$.

En juillet 2017, le ministre russe des Affaires étrangères, Sergueï Lavrov, a effectué une visite à Bruxelles, au cours de laquelle il a rencontré le Premier ministre belge Charles Michel et s'est entretenu avec le vice-premier ministre, le ministre belge des Affaires étrangères Didier Reynders.

L'ancien Premier ministre belge Charles Michel (11 octobre 2014 27 octobre 2019) a déclaré à plusieurs reprises que "les sanctions ne sont pas une fin en soi", qu'elles "sont un moyen de dialogue" et qu'il est favorable à une autre stratégie avec la Russie. "Nous devons maintenir un véritable canal de dialogue avec Moscou, en écouter un autre, quelles que soient les différences" ${ }^{36}$.

Le rôle du facteur russe dans les relations entre l'Ukraine et les pays du Benelux peut également être retracé en analysant l'influence des partis politiques sur la formation de l'opinion publique.

À ce jour, l'influence des partis de droite et d'extrême droite, qui occupent généralement des positions pro-russes aux niveaux national et européen, augmente dans l'UE, et dans les pays du Benelux en particulier. Par exemple, lors des élections européennes de 2014, ces partis ont obtenu des résultats historiquement élevés dans plusieurs pays, dont le Danemark, la France, le Royaume-Uni et les Pays-Bas. La plupart des partis de droite sont opposés à la politique de l'UE, y compris dans le conflit dans l'est de l'Ukraine.

Le Forum du Parti néerlandais pour la démocratie est actuellement actif. Le Forum pour la démocratie a commencé ses activités en 2016, prenant une part active à l'organisation d'un référendum sur la signature de l'accord d'asso- 
ciation entre l'Ukraine et l'Union européenne en 2016. Le parti s'oppose à l'UE et fait campagne pour un référendum sur la sortie du Royaume des PaysBas de l'UE. "Idéologiquement, je suis contre l'UE, contre le marché intérieur, contre l'ouverture des frontières, contre l'euro, contre tout", a déclaré le chef du parti Thierry Bode dans une interview à de Volkskrant. Lors des élections locales (provinciales) de 2019, le Forum pour la démocratie a remporté 86 sièges dans 12 provinces des Pays-Bas. En Hollande méridionale, en Hollande septentrionale et dans le Flevoland, le Forum de la démocratie est devenu le plus grand parti avec 11, 9 et 8 sièges respectivement. Dans toutes les autres provinces, le parti est arrivé en deuxième ou troisième position.

Une analyse distincte devrait être accordée à l'analyse du référendum sur l'accord d'association UE-Ukraine qui s'est tenu aux Pays-Bas le 6 avril 2016. L'initiative référendaire appartient également aux partis de droite des PaysBas, dont le Forum pour la démocratie. Il est intéressant de noter que les politiciens qui ont pris l'initiative d'organiser un référendum aux Pays-Bas ont ouvertement reconnu que l'Ukraine ne les intéressait pas du tout, et leur principal objectif est de retirer les Pays-Bas de l'UE, le soi-disant "Nexit",".

D'une manière générale, l'attention portée à la signature de l'accord d'association avec l'Ukraine est un domaine de discussion distinct. L'UE mène une politique étrangère active, dont l'initiative de partenariat oriental fait désormais partie. Cette initiative a impliqué la participation de six pays d'Europe orientale - l'Ukraine, la Moldavie, la Biélorussie, la Géorgie, l'Arménie et l'Azerbaïdjan. En 2014, dans le cadre du partenariat oriental, des accords d'association avec l'UE ont été signés avec l'Ukraine, la Moldavie et la Géorgie. Alors que les deux derniers accords sont entrés en vigueur en 2016, l'accord d'association UE-Ukraine n'est entré en vigueur qu'en 2017. Pour en revenir au référendum sur l'Ukraine aux Pays-Bas, il est intéressant de se demander pourquoi le référendum n'a eu lieu que sur l'Ukraine, bien qu'un statut spécifique avec L'UE n'était pas prévue pour l'Ukraine.

En Belgique, les partis politiques de la région flamande et de Bruxelles, connus sous le nom de Vlaams Belang, jouent un rôle actif.

Le 26 mai 2019, des élections au Parlement européen, aux parlements fédéral et régional se sont tenues simultanément en Belgique, avec des partis radicaux favorables à l'élargissement des pouvoirs des régions ${ }^{38}$.

En Flandre, le principal parti était la Nouvelle Alliance flamande (N-VA) dirigée par le bourgeois d'Anvers, Bart De Vever. Ce parti représente l'autonomie maximale de la Flandre et le maintien de la plupart des taxes existantes au niveau régional. Cependant, par rapport aux élections de 2014, la performance de l'alliance s'est dégradée de 5\%.

Le Vlaams Belang a remporté la deuxième place en Flandre avec 18,6\% des voix, en hausse de $13 \%$ par rapport aux dernières élections. Cette force 
politique a des opinions encore plus radicales sur l'autonomie économique et politique de la Flandre ${ }^{39}$.

Les partis de droite n'ont pas ignoré le référendum en Crimée. Une organisation non gouvernementale basée en Belgique a invité le Front national français de droite et d'autres partis d'extrême droite, dont la Freiheitliche Partei Österreichs, le FPÖ, l'Intérêt flamand en Belgique et le Parti Jobbik de Hongrie pour surveiller la procédure référendaire du 16 mars 2014 en Crimée. Une équipe d'observateurs a annoncé que le référendum était libre et équitable ${ }^{40}$.

Malgré le renforcement de la position des partis de droite dans certains pays de l'UE, le nombre total de représentants de ces partis au niveau de l'UE est insignifiant par rapport aux principaux partis. Ces parties présentent également des points de vue divergents sur un éventail de questions, les empêchant de se réunir pour faire avancer les vues pro-russes.

L'analyse du rôle du facteur russe dans l'attitude des pays du Benelux envers l'Ukraine de 2014 à 2019 a montré qu'il est extrêmement important à ce stade de comprendre l'influence de la Russie sur les relations entre l'Ukraine et les pays du Benelux. Cette approche permet de déterminer les raisons de la stagnation éventuelle de ces relations et du dialogue entre États.

En général, les stratégies des trois pays du Benelux peuvent être qualifiées de pragmatiques. Cette approche s'explique, tout d'abord, par une coopération étroite dans le domaine de l'économie et les inconvénients d'une rupture complète des relations avec la Russie. Les pays du Benelux sont de petits pays par rapport à leurs voisins européens, dont la principale force est la stabilité économique. Ainsi, pour les pays du Benelux, une intervention active dans le conflit Russie-Ukraine constitue une menace pour les relations économiques.

Dans le même temps, les pays du Benelux soutiennent généralement la politique de sanctions de l'Union européenne contre la Russie, mais insistent sur le dialogue, considérant les sanctions comme un moyen moins efficace d'atteindre les objectifs. Les Pays-Bas ont la position la plus active parmi les pays du Benelux dans le conflit armé russo-ukrainien, cependant, cette position est principalement due à des préoccupations internes et à l'enquête en cours sur la chute du Boeing MH17 sur le territoire ukrainien.

1 Дорошко М. Вплив російського фактора на євроінтеграційний вибір України Економічний часопис - XXI. 2007. № 9-10. C. 46. URL: http://soskin.info/ea/2007/910/200708.html

2 Віднянський С. Європейська політика України: основні етапи, проблеми та перспективи реалізації. Міжнародні зв'язки України: наукові пошуки $i$ знахідки. 2016. Вип. 25. С. 9-40.

${ }^{3}$ The European Union and Russia: Close Neighbours, Global Players, Strategic Partners. URL: http://eeas.europa.eu/archives/docs/russia/docs/russia_brochure07_en.pdf 
${ }^{4}$ Дорошко М. Вплив російського фактора на євроінтеграційний вибір України. С. 46 .

${ }^{5}$ Шеретюк О.М. Політичні імперативи євроінтеграційного курсу України: автореф. дис. ... канд. політ. наук. Київ, 2008. С. 17.

${ }^{6}$ Там само. С. 17.

7 How do European democracies react to Russian aggression? URL: https://www. kremlinwatch.eu/userfiles/how-do-european-democracies-react-to-russian-aggression_ 15273205265116.pdf

${ }^{8}$ Віднянський С., Мартинов А. Україна - Європейський Союз: основні етапи і проблеми співпраці. Україна в історії Європи XIX-nочатку XXI cm.: історичні нариси: монографія. За ред. чл.-кор. НАН України С.В. Віднянського. Київ: Інститут історії України, 2020. С. 662.

${ }^{9}$ Leonard, M., Popescu N. A Power Audit of EU-Russia Relations. The European Council on Foreign Relations. 2007.

${ }^{10}$ Baker M. The Power of Small States: Diplomacy in World War II. University of Chicago Press. 1959.

${ }^{11}$ Olaf F. Knudsen. Small States, Latent and Extant: Towards a General Perspective. Journal of International Relations and Development. 2002.

12 Thorhallsson B. The size of states in the European Union: Theoretical and Conceptual Perspectives. Journal of European Integration. 2006. Vol. 28(1). P. 7-31.

13 Thorhallsson B., Steinsson S. Small State Foreign Policy. The Oxford Research Encyclopedia of Politics (Oxford University Press). 2017.

${ }_{15}^{14}$ Karsh E. Neutrality and small states. Routledge. 1988.

${ }^{15}$ Hirsch M. Luxembourg has learned to defend its own interests in a Europe that increasingly looks like a free-for-all. But it does so with more restraint than others. 2015. URL: https://carnegieeurope.eu/strategiceurope/?fa $=60247$

${ }^{16}$ Interview with Xavier Bettel. We should never forget what Schengen really is. 2015. URL: https://gouvernement.lu/en/gouvernement/xavier-bettel/actualites.gouvernement $\% 2 \mathrm{Bfr}$ $\% 2$ Bactualites\%2Btoutes_actualites\%2Binterviews\%2B2015\%2B08-aout\%2B31-betteleuractiv.html

${ }^{17}$ Luxembourg. URL: https://www.europeanvalues.net/luxembourg/\#_ftn5

18 Liuhto, Kari The economic relations between Luxembourg and Russia. URL: https://www.researchgate.net/publication/323779669_The_economic_relations_between_Luxe mbourg and Russia/citation/download

${ }^{19}$ Larrabee F.S., Pezard S., Radin A., Chandler N., Crane K., Szayna T.S. Russia and the West after the Ukrainian crisis. 2017. URL: https://www.rand.org/content/dam/rand/pubs/ research_reports/RR1300/RR1305/RAND_RR1305.pdf

${ }^{20}$ Dixième session de la commission mixte de coopération économique entre l'union économique belgo-luxembourgeoise et la Russie. URL: https://www.cc.lu/fr/actualites/detail/ xe-session-de-la-commission-mixte-de-cooperation-economique-entre-lunion-economiquebelgo-luxe/

${ }^{21}$ Alfred van Staden: Een trouwe bondgenoot. Baarn 1974, P. 300.

${ }^{22}$ Hans de Boer in WNL Op Zondag on 7 September 2014. URL: https://tvblik.nl/wnl-opzondag/7-september-2014

23 Макаров А.И. Торгово-экономические отношения Российской Федерации и Королевства Нидерландов на современном этапе. Международная торговля и торговая политика. 2018. №2 (14). URL: https://cyberleninka.ru/article/n/torgovo-ekonomicheskieotnosheniya-rossiyskoy-federatsii-i-korolevstva-niderlandov-na-sovremennom-etape

${ }^{24}$ Там само. С. 28. 
${ }^{25}$ Там само. С. 28.

${ }^{26}$ Там само. C. 29.

${ }^{27}$ Kamerbrief over de relaties tussen de EU en Oost-Europa [Parliamentary letter on the relationsbetween the EU and Eastern-Europe]. 12 June 2014f. URL: https://www. rijksoverheid.nl/ministeries/ministerie-van-buitenlandse-53zaken/documenten/kamerstukken/ 2014/06/12/kamerbrief-over-de-relaties-tussende-eu-en-oost-europa

${ }^{28}$ Eilander N. Russian-Dutch relations: Business as unusual Russian and Eurasian Studies. Leiden University. 2017. URL: https://openaccess.leidenuniv.nl/bitstream/handle/1887/49117/ FINAL \%20thesis\%20MA\%20-\%20Nathan\%20Eilander.pdf?sequence $=1$

${ }^{29}$ Haar B. Dutch narratives about Russian-Western relations. 2017. URL: https://www. clingendael.org/sites/default/files/2017-12/Dutch_narratives_about_Russian-Western_ relations.pdf

30 Зовнішня політика України - 2015: стратегічні оцінки, прогнози та пріоритети. За ред. Г.М. Перепелиці. Київ: ВД «Стилос», 2016. С. 118.

${ }^{31}$ Гай-Нижник П.П. Росія проти України (1990-2016 рр.): від політики шантажу і примусу до війни на поглинання та спроби знищення. Київ: «МП Леся», 2017. С. 188.

${ }^{32}$ Renewing the European promise: speech by minister Koenders on the European Union. Speech on the European Union by Mr Bert Koenders, minister of Foreign Affairs of the Kingdom of the Netherlands, Leiden, 30 March 2015. https://www.government.nl/ documents/speeches/2015/03/30/renewing-the-european-promise-speech-by-ministerkoenders-on-the-european-union

${ }^{33}$ Pieters J. "Fear mongering" and "Russia lover": insults fly in debate between right, far right. 2019. URL: https://nltimes.nl/2019/05/23/fear-mongering-russia-lover-insults-fly-debateright-far-righ

${ }^{34}$ Russia: How are trade relations with Belgium? URL: https://www.ing.be/en/retail/mynews/economy/russia-belgium-trade

${ }^{35}$ Speech by Minister Reynders on the priorities of the Belgian diplomacy. 2015. URL: https://diplomatie.belgium.be/en/Newsroom/news/press_releases/foreign_affairs/2015/01/ni_1 90115 speech_minister_reynders

${ }^{36}$ Eeckhaut F. Charles Michel en visite officielle en Russie. 2018. URL: https:// www.rtbf.be/info/belgique/detail_charles-michel-en-visite-officielle-en-russie?id=9822666

37 Barend ter Haar. Dutch narratives about Russian-Western relations. URL: https:// www.clingendael.org/sites/default/files/2017-12/Dutch_narratives_about_Russian-Western_ relations.pdf

${ }^{38}$ Ультраправий лідер очолив парламент Фландрії. URL: https://www.eurointegration. com.ua/news/2019/07/12/7098394/

39 У Бельгії радикальні фламандські партії виграли парламентські і європейські вибори. URL: https://www.eurointegration.com.ua/news/2019/05/27/7096623/

${ }^{40}$ Mitchell A. Orenstein, "Putin's Western Allies". Foreign Affairs. March 25, 2014; Fox, 2014; Andrew Higgins, "Far-Right Fever for a Europe Tied to Russia". New York Times. May 20, 2014.

\section{REFERENCES}

1. Baker, M. (1959). The Power of Small States: Diplomacy in World War II. University of Chicago Press [in English].

2. Dixième session de la commission mixte de coopération économique entre l'union économique belgo-luxembourgeoise et la Russie [10th session of the joint commission for economic cooperation between the Belgian-Luxembourg economic union and Russia]. 
Retrieved from https:/www.cc.lu/fr/actualites/detail/xe-session-de-la-commission-mixte-decooperation-economique-entre-lunion-economique-belgo-luxe/ [in French].

3. Doroshko, M. (2007). Vplyv rosijs'koho faktora na ievrointehratsijnyj vybir Ukrainy [The influence of the Russian factor on Ukraine's European integration choice]. Ekonomichnyj chasopys - XXI, 9-10. Retrieved from http://soskin.info/ea/2007/9-10/200708.html [in Ukrainian].

4. Eeckhaut, F. (2018). Charles Michel en visite officielle en Russie. Retrieved from https://www.rtbf.be/info/belgique/detail_charles-michel-en-visite-officielle-en-russie?id= 9822666 [in French].

5. Federal'naya tamozhennaya sluzhba Rossii [Federal Customs Service of Russia]. Retrieved from http://www.customs.ru [in Russian].

6. Eilander, N. (2017). Russian-Dutch relations: Business as unusual. Russian and Eurasian Studies. Leiden University. Retrieved from https://openaccess.leidenuniv.nl/ bitstream/handle/1887/49117/FINAL\%20thesis\%20MA\%20-\%20Nathan\%20Eilander.pdf? sequence $=1$ [in English]

7. Haar, B. (2017). Dutch narratives about Russian-Western relations. Retrieved from https://www.clingendael.org/sites/default/files/2017-12/Dutch_narratives_about_RussianWestern_relations.pdf [in English].

8. Haj-Nyzhnyk, P.P. (2017). Rosiia proty Ukrainy (1990-2016 rr.): vid polityky shantazhu i prymusu do vijny na pohlynannia ta sproby znyschennia. Kyiv: MP Lesia [in Ukrainian].

9. Hans de Boer in WNL Op Zondag (2014). Retrieved from https://tvblik.nl/wnl-opzondag/7-september-2014_[in Dutch].

10. Hirsch, M. (2015). Luxembourg has learned to defend its own interests in a Europe that increasingly looks like a free-for-all. But it does so with more restraint than others. Retrieved from https://carnegieeurope.eu/strategiceurope/?fa=60247 [in English].

11. How do European democracies react to Russian aggression? Retrieved from https://www.kremlinwatch.eu/userfiles/how-do-european-democracies-react-to-russianaggression_15273205265116.pdf [in English].

12. Interview with Xavier Bettel. (2015). We should never forget what Schengen really is. Retrieved from https:/gouvernement.lu/en/gouvernement/xavier-bettel/actualites. gouvernement $\% 2 \mathrm{Bfr} \% 2 \mathrm{Bactualites} \% 2 \mathrm{Btoutes}$ _actualites\%2Binterviews\%2B2015\%2B08aout\%2B31-bettel-euractiv.html [in English].

13. Kamerbrief over de relaties tussen de EU en Oost-Europa [Parliamentary letter on the relationsbetween the EU and Eastern-Europe]. (2014) Retrieved from https://www. rijksoverheid.nl/ministeries/ministerie-van-buitenlandse-zaken/documenten/kamerstukken/ 2014/06/12/kamerbrief-over-de-relaties-tussende-eu-en-oost-europa [in Dutch].

14. Karsh, E. (1988). Neutrality and small states. Routledge [in English].

15. Larrabee, F.S., Pezard, S., Radin, A., Chandler, N., Crane, K., \& Szayna, T.S. (2017). Russia and the West after the Ukrainian crisis. Retrieved from https://www.rand.org/content/ dam/rand/pubs/research_reports/RR1300/RR1305/RAND_RR1305.pdf [in English].

16. Leonard, M. \& Nicu P. (2007). A Power Audit of EU-Russia Relations. The European Council on Foreign Relations [in English].

17. Liuhto, K. (2018). The economic relations between Luxembourg and Russia. Retrieved from https://www.researchgate.net/publication/323779669_The_economic relations_between_Luxembourg_and_Russia/citation/download [in English]. English].

18. Luxembourg. Retrieved from https://www.europeanvalues.net/luxembourg/\#_ftn5 [in

19. Makarov, A.Y. (2018). Torhovo-ekonomycheskye otnoshenyia Rossyjskoj Federatsyy y Korolevstva Nyderlandov na sovremennom etape [Trade and economic relations of the 
Russian Federation and the Kingdom of the Netherlands at the present stage]. Mezhdunarodnaia torhovlia y torhovaia polytyka - International trade and trade policy. № 2 (14). Retrieved from https://cyberleninka.ru/article/n/torgovo-ekonomicheskie-otnosheniyarossiyskoy-federatsii-i-korolevstva-niderlandov-na-sovremennom-etape [in Russian].

20. Olaf F. Knudsen (2002). Small States, Latent and Extant: Towards a General Perspective. Journal of International Relations and Development [in English].

21. Orenstein, M.A. (2014). Putin's Western Allies. Foreign Affairs [in English].

22. Perepelytsia, H.M. (Eds.). (2016). Zovnishnia polityka Ukrainy - 2015: stratehichni otsinky, prohnozy ta priorytety. Kyiv: VD Stylos [in Ukrainian].

23. Pieters, J. (2019). "Fear mongering" and "Russia lover": insults fly in debate between right, far right. Retrieved from https://nltimes.nl/2019/05/23/fear-mongering-russia-loverinsults-fly-debate-right-far-righ [in English].

24. Renewing the European promise: speech by minister Bert Koenders on the European Union (2015). Retrieved from https://www.government.nl/documents/speeches/2015/03/30/ renewing-the-european-promise-speech-by-minister-koenders-on-the-european-union [in English].

25. Russia: How are trade relations with Belgium? (2018). Retrieved from https://www. ing.be/en/retail/my-news/economy/russia-belgium-trade [in English].

26. Sheretiuk, O.M. (2008). Politychni imperatyvy ievrointehratsijnoho kursu Ukrainy. [Political imperatives of Ukraine's European integration course]. Extended abstract of PhD thesis. Kyiv [in Ukrainian]

27. Speech by Minister Reynders on the priorities of the Belgian diplomacy. (2015). Retrieved from https://diplomatie.belgium.be/en/Newsroom/news/press_releases/foreign_ affairs/2015/01/ni_190115_speech_minister_reynders [in English].

28. Staden, A. (1974). Een trouwe bondgenoot: Nederland en het Atlantische Bondgenootschap (1960-1971) [A loyal ally: the Netherlands and the Atlantic Alliance (1960 1971)]. Anthos, Baarn [in Dutch].

29. The European Union and Russia: Close Neighbours, Global Players, Strategic Partners. Retrieved from http://eeas.europa.eu/archives/docs/russia/docs/russia_brochure 07 en.pdf [in English].

30. Thorhallsson, B. \& Steinsson, S. (2017). Small State Foreign Policy. The Oxford Research Encyclopedia of Politics (Oxford University Press) [in English].

31. Thorhallsson, B. (2006). The size of states in the European Union: Theoretical and Conceptual Perspectives. Journal of European Integration, 28(1) [in English].

32. Togt, T. (2015). How should Europe respond to Russia? The Dutch view. Retrieved from https://www.ecfr.eu/article/commentary_how_should_europe_respond_to_russia_the dutch_view311233 [in English].

33. U Belhii radykalni flamandski partii vyhraly parlamentski i ievropejski vybory [In Belgium, radical Flemish parties have won parliamentary and European elections]. (2019). Retrieved from https://www.eurointegration.com.ua/news/2019/05/27/7096623/ [in Ukrainian].

34. Ultrapravyj lider ocholyv parlament Flandrii [The far-right leader has led the Flanders Parliament] (2019). Retrieved from https://www.eurointegration.com.ua/news/2019/07/12/ 7098394/ [in Ukrainian].

35. Vidnyanskyj, S. (2016). Yevropejs'ka polityka Ukrainy: osnovni etapy, problemy ta perspektyvy realizatsii. Mizhnarodni zv'iazky Ukrainy: naukovi poshuky i znakhidky International Relations of Ukraine: Scientific Searches and Findings, 25, 9-40 [in Ukrainian].

36. Vidnyanskyj, S., \& Martynov, A. (2020). Ukraina - Yevropejs'kyj Soiuz: osnovni etapy i problemy spivpratsi. Ukraina $v$ istorii Yevropy XIX - pochatku XXI st.: istorychni narysy: monohrafiia. Kyiv [in Ukrainian]. 